Article

\title{
Investigating Characteristics of the Static Tri-Switches Tactile Probing Structure for Micro-Coordinate Measuring Machine (CMM)
}

\author{
Yin Tung Albert Sun ${ }^{1}$, Kuo-Yu Tseng ${ }^{2}$ and Dong-Yea Sheu ${ }^{1, *}$ \\ 1 Graduate Institute of Manufacturing Technology, National Taipei University of Technology, Taipei 10608, \\ Taiwan; ytsun@mail.ntut.edu.tw \\ 2 Micro Machining Laboratory, National Taipei University of Technology, Taipei 10608, Taiwan; \\ andy80618@yahoo.com.tw \\ * Correspondence: dongyea@ntut.edu.tw; Tel.: +886-2-2771-2171
}

Academic Editor: Kuang-Cha Fan

Received: 1 April 2016; Accepted: 29 June 2016; Published: 13 July 2016

\begin{abstract}
This paper describes the fabrication of a series of micro ball-ended stylus tips by applying micro-EDM (Electrical Discharge Machining) and OPED (One Pulse Electrical Discharge) processes, followed by a manual assembly process of a static tri-switches tactile structure on a micro-CMM (Coordinate Measuring Machine). This paper further proves that the essential performance of the proposed system meets an acceptable benchmark among peer micro-CMM systems with a low cost. The system also adjusts for ambient temperature and humidity as the ordinary lab environmental conditions. For demonstration, several experiments used a randomly selected glass stylus with the diameters of stem and sphere of $0.07 \mathrm{~mm}$ and $0.12 \mathrm{~mm}$, respectively. By leveraging research guidelines and common practice, this paper further investigates the probing relationship between measurement accuracy and its associated critical characteristics, namely triggering scenarios and geometric feature probing validation. The experimental results show that repeated detections in the uncertainty, in vertical and horizontal directions of the same point, achieved as small as $0.11 \mu \mathrm{m}$ and $0.29 \mu \mathrm{m}$, respectively. This customized tri-switches tactile probing structure was also capable of measuring geometric features of micro-components, such as the inner profile and depth of a micro-hole. Finally, extensions of the proposed approach to pursue higher accuracy measurement are discussed.
\end{abstract}

Keywords: micro-EDM; micro spherical stylus tip; micro-CMM

\section{Introduction}

Thanks to ongoing advancements in micro manufacturing technology over the past several decades, demand for micro-products, such as micro bio-medical and optical devices and MEMS (Microelectromechanical Systems) products, has been increasing significantly. To enhance manufacturing efficiency and improve quality for micro-products, high accuracy measuring devices are essential for micro fabrication technology. Conventional measuring devices, such as the Vernier calipers and micrometers, are incapable of measuring delicate micro-components. While a wide variety of optical measuring methods have been developed for measurement of micro-products, non-contact methods have yet to be developed for measuring the lateral-wall, high-aspect ratio and high reflection in micro-parts. More recently, in order to measure the geometry of micro-products, the micro coordinate measuring machine $(\mu-\mathrm{CMM})$ and a number of delicate tactile triggering structures have been developed [1-3]. However, the dimension of micro spherical stylus tips is one of the critical challenges for micro-CMMs. Although the micro tactile spherical styli with a diameter of $0.125 \mathrm{~mm}$ are 
commercially available, they are sold less often. To utilize a spherical stylus of such a small dimension, a micro-CMM with a high sensitive probing structure is necessary [3]. Due to the limitations of the stylus dimension, micro-CMMs still face some challenges when measuring the geometry of micro-components, such as micro-holes or micro-slots with a high aspect ratio. Micro ball-ended stylus tips, accomplished by a glue assembly process, held great precision with an eccentric deviation from $0.6 \mu \mathrm{m}$ to $1 \mu \mathrm{m}$ [4]. In our previous study, OPED (One Pulse Electrical Discharge) and gluing processes were successful to fabricate micro spherical stylus tips with a diameter of less than $0.1 \mathrm{~mm}$ [5]. To keep the strength of adherence sufficient for the micro-triggering touch, a Wire Electrical Discharge Grinding (WEDG) process was successful in producing micro stylus tips with diameters of less than $0.15 \mathrm{~mm}$ and the roundness of less than $1 \mu \mathrm{m}$, respectively, for the purpose of controlling the amount of glue applied between the glass ball and the stem [6].

The other critical problem with the micro-CMM is a sensing structure system. In the contact measuring, the stylus tip makes contact with the micro-object to trigger an electric signal. At the same time, the impact force is generated instantaneously. There is a correlation of size and fragility being the smaller the stylus, the more fragile it is. In our previous study, a micro stylus tip with a $40 \mu \mathrm{m}$ stem diameter fractured when the impact force exceeded $11 \mathrm{mN}$ [7]. To prevent them from breaking and fracturing easily during the measuring process, the sensing structure should have a sufficient stiffness in all direction [8]. Alblalaihid et al. applied $1 \mathrm{mN}$ contact force to assess variable stiffness in the $z$-direction [9]. In addition, previous researchers reported various tactile sensing mechanisms of micro-CMM probing heads. However, these delicate triggering structures depended upon MEMS processes $[10,11]$, which makes the probing heads so costly that they are not commercially available in the market. Our previous study reported a tri-switches tactile probing structure, focusing on only the vertical directional tactile triggering and few applications [12]. Subsequently, our other previous work enhanced both fabrication of micro-styli and multi-directional tactile triggering structure, facilitating the exploration of diverse measurement strategy [13]. Through system advancements, Alblalaihid et al. defined an associated measurement strategy for a specific probe $[14,15]$ with stiff and flexible modes. It is observed that such an integral strategy is customized to a developed micro-CMM system while some benchmark parameters associated with the triggering scenarios are affected by the stiffness of a micro-probe, and are transferrable among micro-CMMs.

Conclusions drawn from the heretofore-mentioned systems provide segregated insight about the variable triggering scenario through a surface interaction to affect measurement accuracy, which is critical to the performance of micro-CMMs. Therefore, this paper not only refers to previously approved research guidelines and sound hands-on practice with a lean development in mind, but also investigates the probing relationship between measurement accuracy and critical characteristics such as triggering scenarios and geometric feature probing validation.

\section{Materials and Methods: Probing Head Fabrication and Assembly}

Because different measuring effects on the probed geometry stem from hardware construction, this section categorizes three aspects of stylus related fabrication.

\subsection{Principle of Tri-Switches Probing Head Triggering Mechanism}

To achieve a low-cost but high-sensitivity structure, the research team developed an enhanced sensing structure and tactile detecting mechanism from the previous work, as shown in Figure 1, to overcome the previously mentioned disadvantages and to achieve multi-directional measurement. Three micro wires of $50 \mu \mathrm{m}$ in diameter were used to support the circle plate with 120 degrees apart. Three sensing rods of $150 \mu \mathrm{m}$ in diameter were fixed upon the micro wires in each orthogonal direction. A micro spherical stylus tip was attached onto the circle plate using glue. The micro wires and rods maintain normal point-contacts in each direction when the stylus tip is in a standby condition. This means that the tri-switches of the micro stylus tip normally keeps the circuits closed without touching anything. However, when the stylus tip touches a micro-product, the three 
point-contacts switch to non-contact status instantly, and three detecting voltages change significantly. The micro-CMM system that was equipped with the proposed triggering mechanism of the tri-switches probing head, shown in Figure 2, used the electronic signal, to detect any reachable positions through triggering actions.

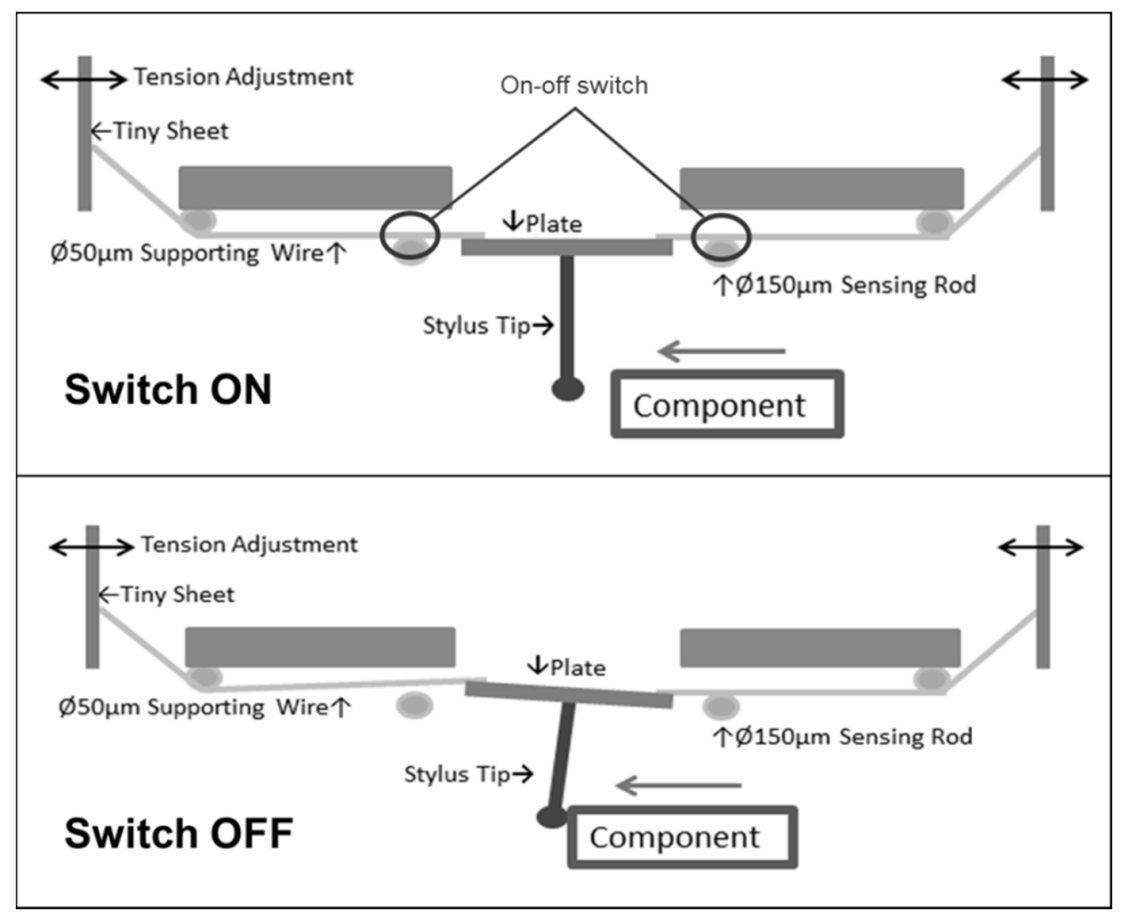

Figure 1. Tactile triggering mechanism of the proposed tri-switches probing head.

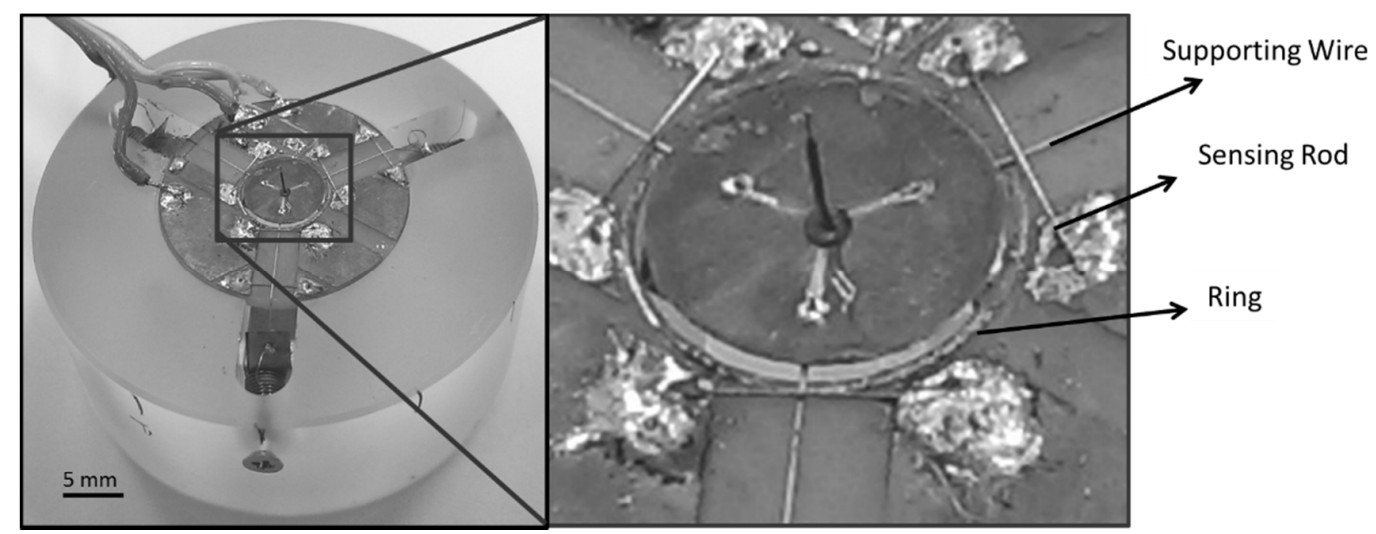

Figure 2. Tri-switches tactile probing structure.

\subsection{Spherical Stylus Tips Fabrication}

Micro spherical stylus tips play an important role in micro-CMM tactile probing heads. To detect the micro-component surface more precisely, the micro spherical stylus tips with a diameter less than $0.15 \mathrm{~mm}$ are necessary. In order to fabricate micro stylus tips, an assembly process called Wire Electrical Discharge Grinding (WEDG) and the OPED process were utilized. On the other hand, with properly setup parameters, the OPED process produced tungsten spherical stylus tips with consistently high precision. The scale of the aforementioned styli, as shown in Figure 3a,b, was suitable for the tri-switches probing head assembly detailed in the following section. In order to investigate the relationship between measurement accuracy and comprehensive characteristics, namely triggering 
scenarios and feature probing validation, a randomly selected spherical stylus was manually assembled onto the CMM probing head. In addition, the diameters of stem and sphere for the studied glass stylus were $0.07 \mathrm{~mm}$ and $0.12 \mathrm{~mm}$, respectively, as shown in Figure 3b. The confirmatory results in Section 3 are applicable and representative for overall stylus tips mentioned in terms of the realistic measuring behavior of the constructed probing head in this study.

(a)

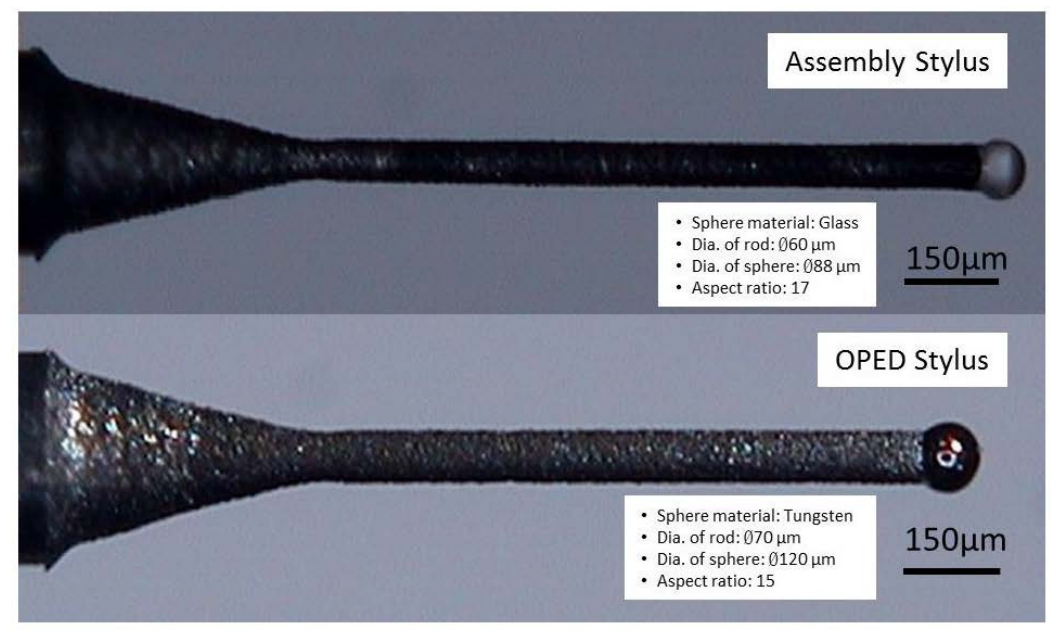

Figure 3. Stylus tips produced by an (a) assembly process called WEDG (Wire Electrical Discharge Grinding) process and the (b) OPED (One Pulse Electrical Discharge) process.

\subsection{Probing Head Assembly Process}

In order to assemble the micro spherical stylus tip onto the circle plate manually, two sets of $x-y$ stages and one CCD (Charge Coupled Device) camera were used for position alignment, as shown in Figure 4.

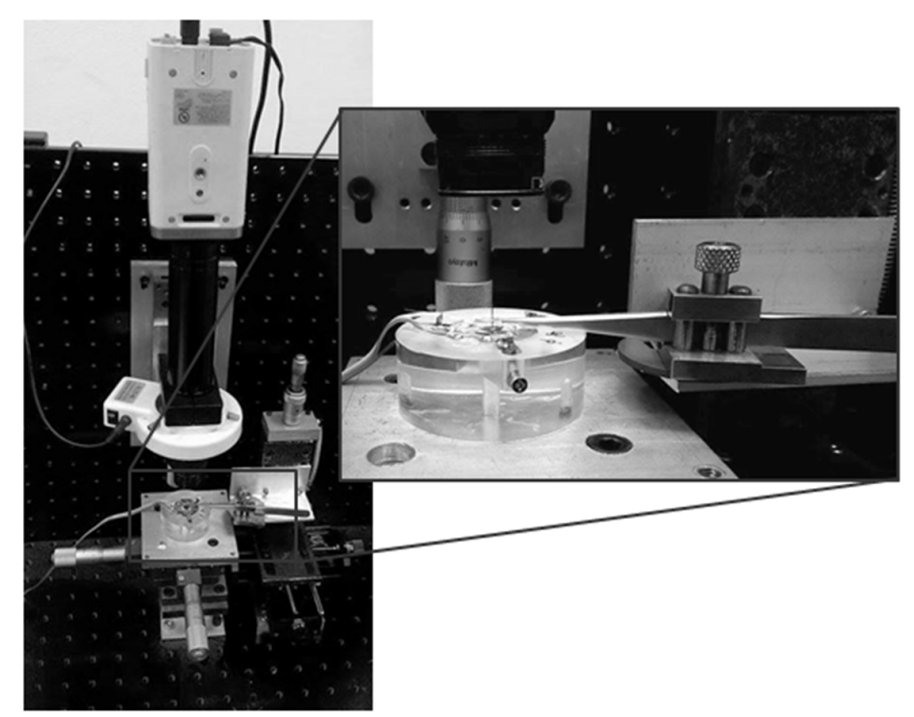

Figure 4. Manual assembly process for the tri-switches probing head.

Most commercial micro-CMMs are unable to use this tri-switches probing structure due to problems of compatibility and the extremely tiny stylus tip. To investigate measuring behaviors and evaluate the tri-switches probing structure, a three-axis high-resolution machine similar to the micro-CMM was set up, as shown in Figure 5a. This micro-CMM was controlled by a PC running a LabVIEW program (Version 11.0, National Instruments, Austin, TX, USA), as shown in Figure 5b. 
A laser interferometer was used to calibrate position accuracy of this similar micro-CMM; the deviation of three-axis position accuracy was within the range of $\pm 3 \mu \mathrm{m}$. The tri-switches probing structure was mounted onto this micro-CMM to analyze its triggering behaviors. The micro-CMM, however, is clearly insufficient for high nano-order accuracy metrology. This research also focused on investigating the triggering possibility of the proposed probing head. The accuracy of this micro-CMM has improvement potential for high-accuracy measurement in the future.

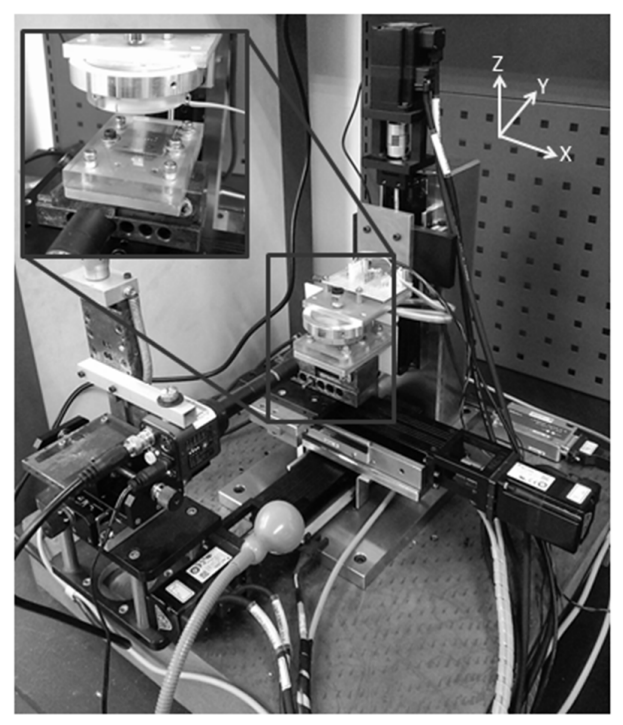

(a)

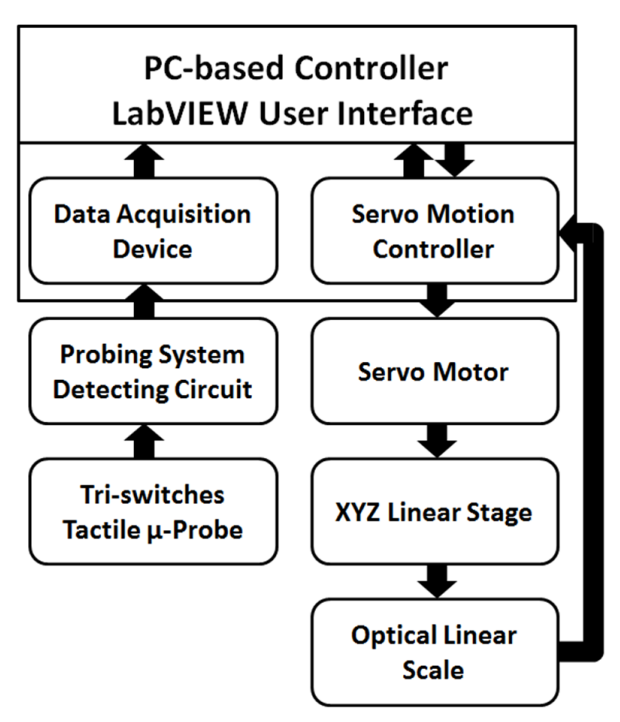

(b)

Figure 5. Scheme (a) and overview (b) of the developed micro-CMM (Coordinate Measuring Machine) system.

\section{Results: Summarized Characteristics of Triggering Scenarios and Probed Feature Validation}

The experiments involved two major investigations: (1) the triggering force, mechanism structure, and directional behaviors of the stylus; and (2) the measurement validation of the probed geometric features.

\subsection{Triggering Force}

In order to investigate the impact by a surface interaction, the triggering force of the tri-switches probing head was measured by a micro load cell which was mounted on the micro-CMM to measure the triggering force in the $z$-axis direction. After 30 contact repetitions, the average triggering force in the vertical direction was estimated as $1.27 \pm 0.01 \mathrm{mN}$ with $5 \%$ of risk, which met the stiffness requirement of the probe in all three directions and the isotropy of the probing force for triggering. Therefore, the micro stylus tip was able to endure the aforementioned impact force during the measuring process.

\subsection{Mechanism Structure}

The probing performance is dependent upon the mechanism structure, including the length of the micro stylus tip and the tension strength of the micro-wires. To measure micro-components with a high aspect ratio, the micro-CMM requires a longer stylus tip, as the sensitivity of tri-switches probing structure is determined by stylus tip length. In this study, two stylus tips consisting of a stainless steel plate and the electrical sensing module with the stem lengths of 5 and $10 \mathrm{~mm}$ were used to investigate triggering behaviors. While the performance of triggering behaviors on the $z$-axis with these two stylus tips are theoretically identical, triggering accuracy is compromised in the horizontal direction, as shown in Figure 6. To prevent the undesirable elastic deformation of the bending stylus impeding the triggering accuracy, the stylus of $5 \mathrm{~mm}$ was used. Although using a finite element model (FEM) to 
study the relationship between applied sensing load and probe stiffness is not within the scope of the study, a trend of using FEM facilities has led to a similar development approach [15].

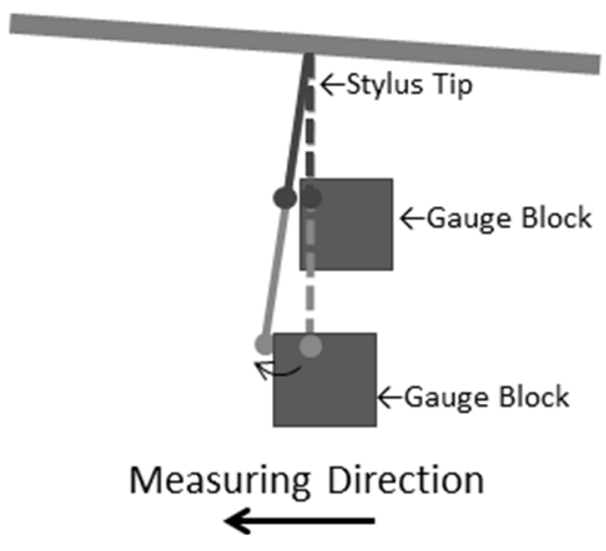

Figure 6. Avoiding the elastic deformation of stem bending which creates an undesirable triggering behavior.

\subsection{Triggering Behaviors in Vertical and Lateral Directions}

Following completion of the probing head assembly, the triggering characteristics of this probing structure on the same point were investigated through repeated detecting trials. For the vertical direction, as shown in Figure 7, the detection of the z-axis triggering position resulted in detection uncertainty to an appropriate extent. Therefore, tri-switches probing structure was capable of measuring geometry profile with micro-scale height difference in the vertical direction. Due to the cost associated with uncertainty test referring to both types A and B with reference to GUM (Guide to Uncertainty in Measurement): resolution of the instruments, temperature variation, variation in position detection, etc. was consolidated into data collection performed by common practice.

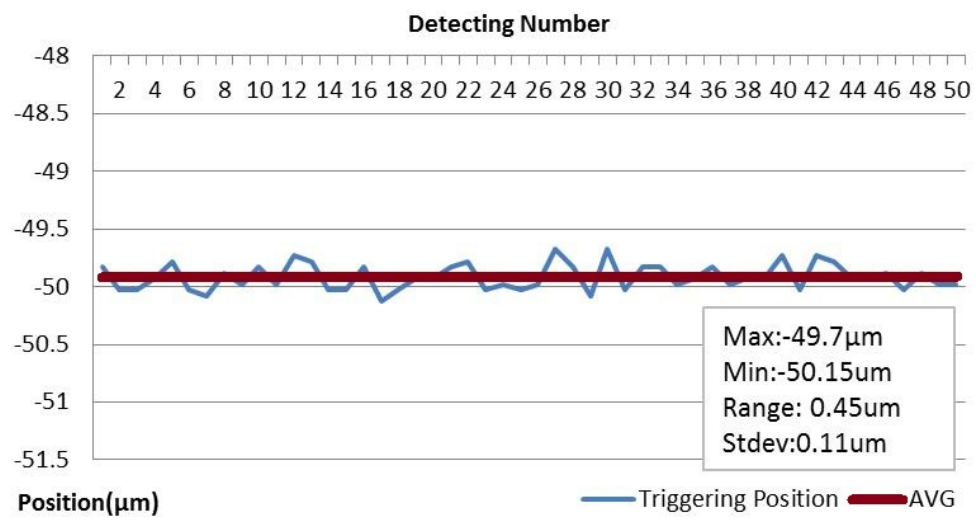

Figure 7. Detection uncertainty in the vertical z-axis direction.

As for the horizontal direction, the lateral triggering characteristics of this probing structure on the same point were investigated through repeated detecting trials in predetermined six angulated divisions, as illustrated in Figure 8. The results about the uncertainty appeared less than $1 \mu \mathrm{m}$ in nine out of twelve angle graduations, shown in Figure 9. Although the deviation of the lateral triggering behaviors at 90,120, and 150 degree graduations were relatively larger than the rest, the uncertainty enhancement compared to the former model [12] was evident. 

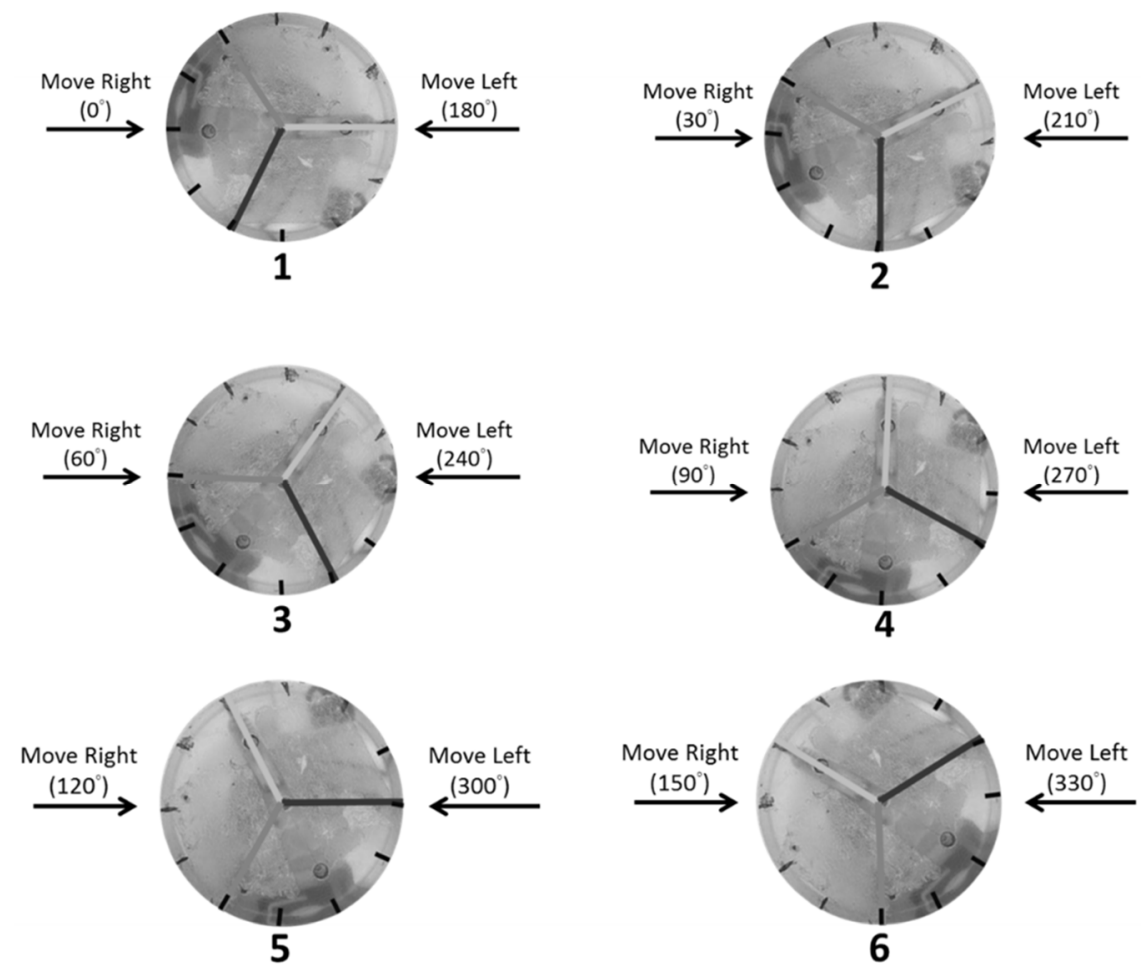

Figure 8. Lateral triggering measurements in six angulated divisions.

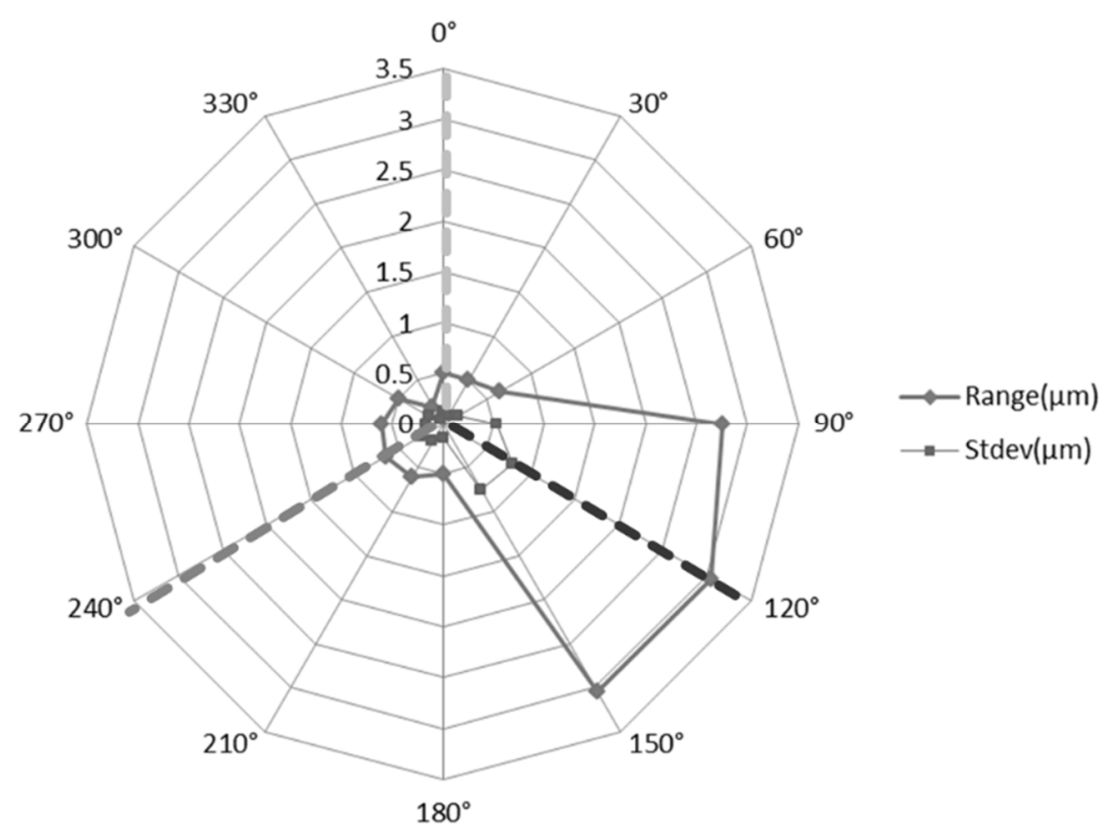

Figure 9. Lateral triggering detection uncertainty.

Such a behaving scenario most likely stemmed from the unbalanced tension strength of the supporting wires, which were incapable of recovering their initial positions. As an improvement, adding an auxiliary sensing wire structure may resolve the issue to achieve higher accuracy measurements. 


\subsection{Surface Interaction on Different Materials of Spheres}

In dealing with probing head systems in the order of micrometers, the phenomenon of surface interaction needs to be considered. The effect of surface interaction shows rising significance and cannot be disregarded, especially when using a smaller-scale stylus tip to measure micro-products.

Two types of processes were applied to fabricate micro spherical stylus tips in this study. The materials of the sphere were glass and tungsten in the assembly process and the OPED process, respectively. Two stylus tips with sphere material were used to investigate measuring behaviors on different material within the component. The component is a gauge block made of metal and a spherical LED (Light-emitting Diode) lens made of resin, respectively. A sticking force can be observed when the micro sphere comes off of the feature surface during horizontal direction measurement. The experimental results showed that the glass sphere produced a significant oscillation that might impact a sensing wire structure causing measurement deviation, as shown in Figure 10. Although sticking force between metal sphere and spherical LED resin was observed to be extremely minute, with virtually no oscillation when measuring the gauge block with a metal sphere, the potential influence of the static electricity caused by insufficient isolation about remnant (static) charges between the gauge block and the sphere should be investigated in the future extended study. Because of the oscillation phenomenon and sufficient hardness, this study utilized the WEDG and OPED processes to fabricate glass and tungsten micro spherical stylus tips, respectively.

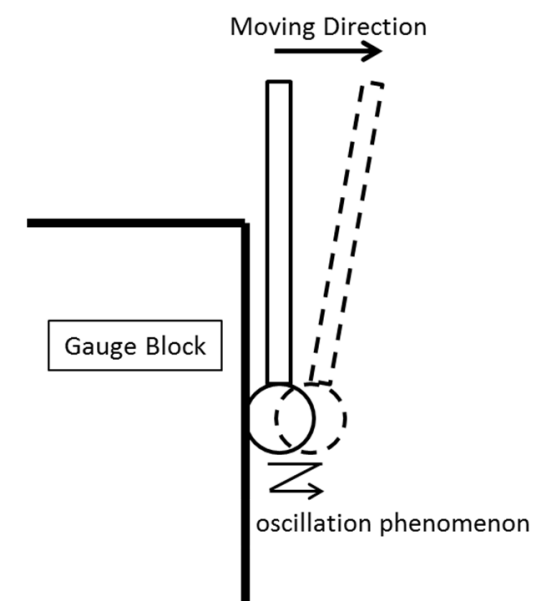

Figure 10. Oscillation phenomenon occurred while measuring.

\subsection{Geometric Profile Measurement}

In this study, the tri-switches probing structure was used in several measuring examples. Measuring the step between two different gauge blocks illustrated one case. One of the critical problems in precision measurement was temperature, since maintaining a steady temperature in the environment tended to be difficult. This study used the same material gauge blocks that had similar thermal expansion coefficients in order to decrease deviation. These two gauge blocks were mounted on a granite platform, as shown in Figure 11. The results showed that the respective averages of the step were $252.45 \mu \mathrm{m}$ and $253.83 \mu \mathrm{m}$ in the vertical direction and 0 degree in a lateral direction. The deviations were caused by many factors, including machines, programs, calibration, environments, and probing heads. 


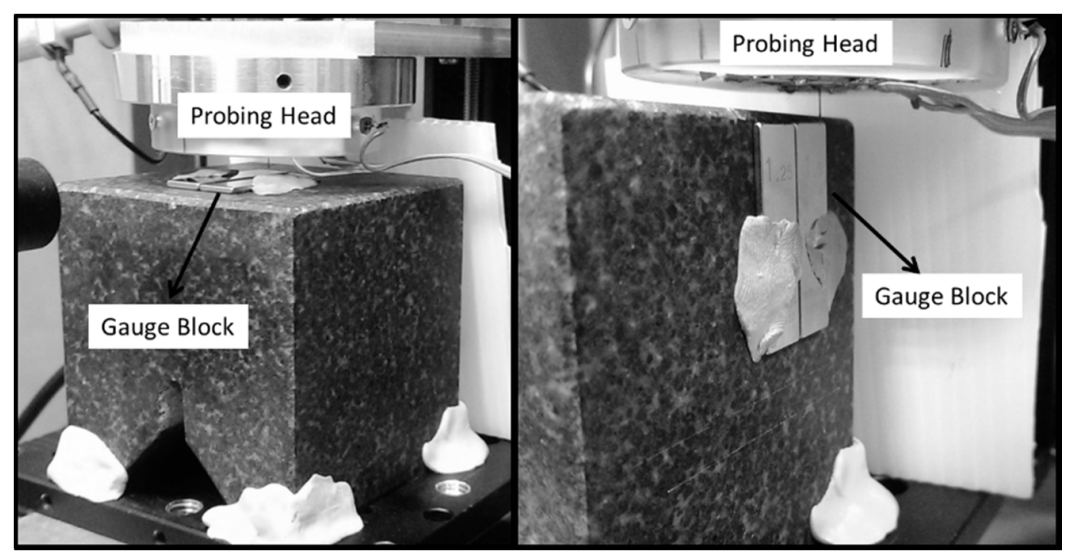

Figure 11. Gauge blocks mounted on the granite platform.

\subsection{Micro-Component Geometric Feature Measurement}

Two kinds of micro-component geometric features, namely hole profile and depth, were studied to validate the performance of the micro-probing structure. Precision geometric measurement technologies have been under development for decades. In the case of micro-components with high aspect ratios, high optical-reflection and high curvature surfaces, however, micro-CMMs are still more reliable than an optical metrology device, particularly because these optical metrology devices are still incapable of precisely measuring the geometry profile of an inside micro-hole with a diameter of less than $0.2 \mathrm{~mm}$. Nevertheless, these micro-holes with high aspect ratios are widely applied to engine nozzles, printer nozzles and wire drawing dies utilized in the textile industry. In this study, therefore, the inside geometry profile of the micro-hole was measured by the proposed tri-switches probing structure. The experiment drove the probe to collect hits across numerous depths in discrete movements with an upward increment of $5 \mu \mathrm{m}$. The profile detecting results were consistent with the inner geometry of the micro-hole according to the section observation of the optical metrology device, as shown in Figure 12.
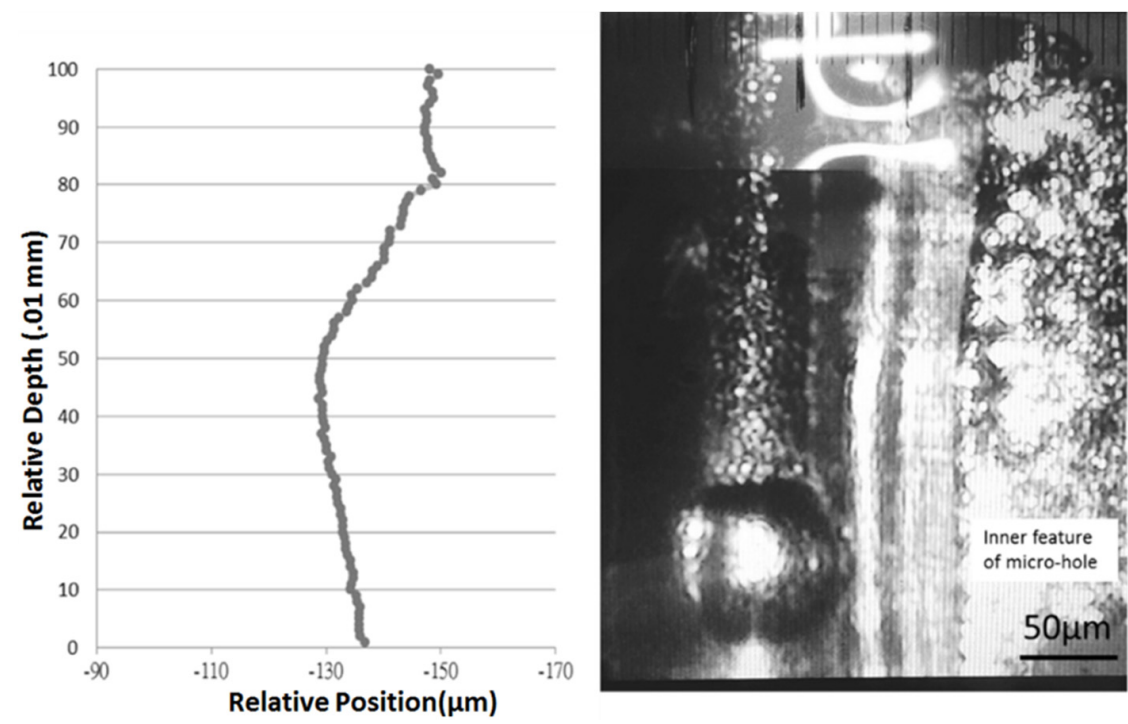

Figure 12. Consistency of measuring the inner profile of a micro-hole: the proposed vs. optical device.

The depth of micro-holes in this study was measured by the tri-switches probing head. The depth of micro-holes drilled by a $\varnothing 0.3 \mathrm{~mm}$ micro drilling tool could be easily measured by a spherical stylus tip with sphere diameter of $\varnothing 120 \mu \mathrm{m}$, as shown in Figure 13. 


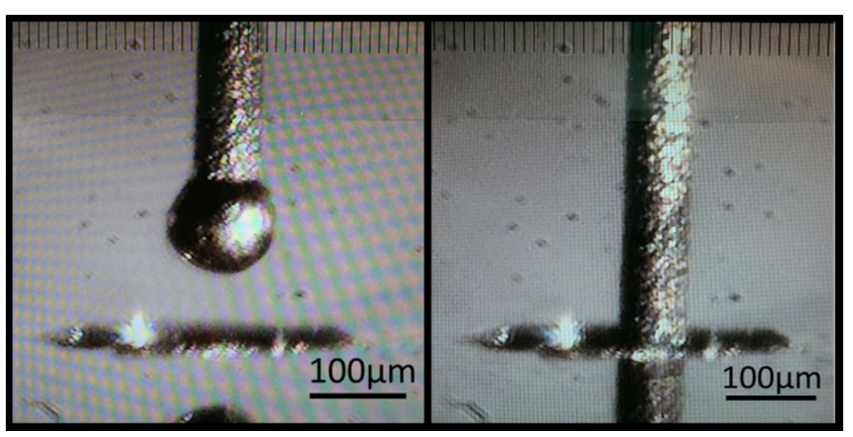

Figure 13. Depth of a micro-hole being measured by the probing head.

High-accuracy performance in the vertical direction of the tri-switches probing head was also validated with this experimental result, which involved 30 repeated measurements of the depth of a micro-hole. The average depth was estimated $222.82 \pm 0.20 \mu \mathrm{m}$ with $5 \%$ risk. However, compared to the results from using an optical microscope (the within uncertainty was close to $20 \mu \mathrm{m}$ ), as shown in Figure 14, the differential value was as much as $15.43 \mu \mathrm{m}$. It might be due to the lip clearance angle of the micro drilling tool. Because the inside bottom of the micro-hole was not leveled, measurement results by these two devices tended to be inconsistent. Otherwise, the difference between them could be more ideal. Therefore, the height feature that was measured by this micro-CMM system maintained more favorable reproducibility than the optical metrology device.

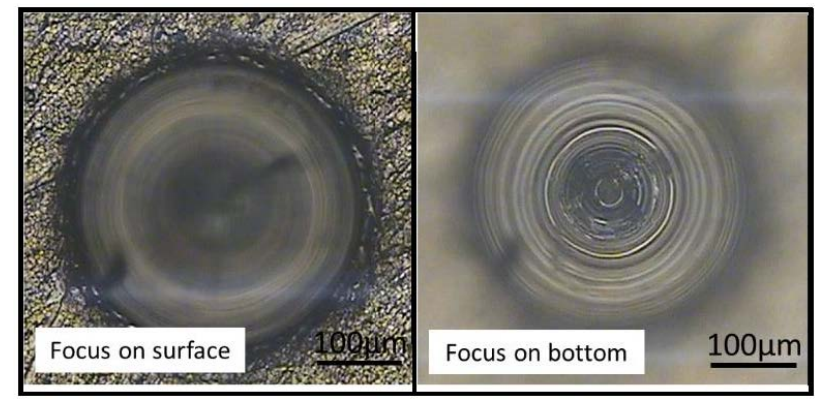

Figure 14. Measurement results by optical microscope.

\section{Discussion}

The technology introduced in this paper combines fabrication and assembling processes to develop a novel probing head that is mounted on a micro-CMM and is capable of multi-directional measurement on geometric features of a micro-part. As explained, the resulting sensing structure and characteristics by this tri-switches probing head structure indicate how it will be possible to measure micro-parts.

The tactile probing structure for micro tri-switches is composed of a distinctive sensing wire structure and an assembled micro spherical stylus tip, in which the diameters of the stem and sphere are $0.07 \mathrm{~mm}$ and $0.12 \mathrm{~mm}$, respectively. In experiments on the same point with repeated detecting, repeatability was as low as $0.11 \mu \mathrm{m}$ in the vertical direction. Repeatability deviation and triggering displacement in a lateral direction was less than $3 \mu \mathrm{m}$ and $50 \mu \mathrm{m}$, respectively. In experiments measuring inside geometry profiles of micro-holes, the results proved that the developed tri-switches probing structure was practicable for measuring the surface features of micro-components. In lateral measurement, the effect of unbalanced tension strength from supporting wires and improper position of the sensing rod could possibly influence triggering behaviors. Therefore, to achieve higher accuracy measurement, future improvements may include an auxiliary sensing wire structure in order to take advantage of the low-cost static tri-switches triggering structure for measuring the geometry and 
profiles of micro-products. As for the observed sticking force, isolations from remnant static electricity, van der Waals force, air currents, temperature variation and external vibrations, to name a few, should be considered in future system evaluations. In addition, using FEM facilities to predict the mechanical deformation of all mechanisms under load is also part of the extended future research work.

Author Contributions: Dong-Yea Sheu and Yin Tung Albert Sun conceived and designed the experiments; Kuo-Yu Tseng performed the experiments; Yin Tung Albert Sun and Dong-Yea Sheu analyzed the data; Yin Tung Albert Sun and Kuo-Yu Tseng wrote the paper.

Conflicts of Interest: The authors declare no conflict of interest.

\section{References}

1. Küng, A.; Meli, F.; Thalmann, R. Ultraprecision micro-CMM using a low force 3D touch probe. Meas. Sci. Technol. 2007, 18, 319-327. [CrossRef]

2. Claverley, J.D.; Leach, R.K. A vibrating micro-scale CMM probe for measuring high aspect ratio structures. Microsyst. Technol. 2010, 16, 1507-1512. [CrossRef]

3. Bos, C. Aspects of tactile probing on the micro scale. Precis. Eng. 2011, 35, 228-240. [CrossRef]

4. Hansen, H.N.; Carneiro, K.; Haitjema, H.; De Chiffre, L. Dimensional micro and nano metrology. CIRP Ann.-Manuf. Technol. 2006, 55, 721-743. [CrossRef]

5. Cheng, C.C.; Huang, J.K.; Sheu, D.Y. Development of gluing and assembling process on micro EDM to fabricate micro spherical stylus tips for micro-CMM. In Proceedings of the 6th International Conference on MicroManufacturing ICOMM, Tokyo, Japan, 7-10 March 2011; pp. 219-222.

6. Sheu, D.Y.; Cheng, C.C. Assembling ball-ended styli for CMM's tactile probing heads on micro EDM. Int. J. Adv. Manuf. Technol. 2013, 65, 485-492. [CrossRef]

7. Sheu, D.Y. Micro-spherical probes machining by EDM. J. Micromech. Microeng. 2004, 15, 185-189. [CrossRef]

8. Fan, K.C.; Cheng, F.; Wang, H.Y.; Ye, J.K. The system and the mechatronics of a pagoda type micro-CMM. Int. J. Nanomanuf. 2012, 67-86. [CrossRef]

9. Alblalaihid, K.; Kinnell, P.; Lawes, S. Fabrication and characterisation of a novel smart suspension for micro-CMM probes. Sens. Actuators A: Phys. 2015, 232, 368-375. [CrossRef]

10. Brand, U.; Kleine-Besten, T.; Schwenke, H. Development of a special CMM for dimensional metrology on microsystem components. In Proceedings of the 15th Annual Meeting of the American Society for Precision Engineering, Scotsdate, AZ, USA, 22-27 October 2000; Volume 542, pp. 1-5.

11. Claverley, J.D.; Sheu, D.Y.; Burisch, A.; Leach, R.K.; Raatz, A. Assembly of a novel MEMS-based 3D vibrating micro-scale co-ordinate measuring machine probe using desktop factory automation. In Proceedings of the 2011 IEEE International Symposium on Assembly and Manufacturing (ISAM), Tampere, Finland, 25-27 May 2011.

12. Kao, S.M.; Sheu, D.Y. Developing a novel tri-switch tactile probing structure and its measurement characteristics on micro-CMM. Measurement 2013, 46, 3019-3025. [CrossRef]

13. Sheu, D.Y. Manufacturing tactile spherical stylus tips by combination process of micro electro chemical and one-pulse electro discharge technology. Int. J. Adv. Manuf. Technol. 2014, 74, 741-747. [CrossRef]

14. Alblalaihid, K.; Kinnell, P.; Lawes, S.; Desgaches, D.; Leach, R. Performance assessment of a new variable stiffness probing system for micro-CMMs. Sensors 2016, 16, 492. [CrossRef] [PubMed]

15. Alblalaihid, K.; Lawes, S.; Kinnell, P. Variable stiffness probing systems for micro-coordinate measuring machines. Precis. Eng. 2016, 43, 262-269. [CrossRef]

(C) 2016 by the authors; licensee MDPI, Basel, Switzerland. This article is an open access article distributed under the terms and conditions of the Creative Commons Attribution (CC-BY) license (http://creativecommons.org/licenses/by/4.0/). 